\title{
An experimental investigation of the internal magnetic field topography of an operating Hall thruster
}

\author{
Peter Y. Peterson, ${ }^{\text {a) }}$ Alec D. Gallimore, and James M. Haas \\ Plasmadynamics and Electric Propulsion Laboratory, Department of Aerospace Engineering, \\ The University of Michigan, College of Engineering, Ann Arbor, Michigan 48109
}

(Received 1 May 2002; accepted 25 July 2002)

\begin{abstract}
Magnetic field measurements were made in the discharge channel of the $5 \mathrm{~kW}$-class P5 laboratory-model Hall thruster to investigate what effect the Hall current has on the static, applied magnetic field topography. The P5 was operated at 1.6 and $3.0 \mathrm{~kW}$ with a discharge voltage of 300 $\mathrm{V}$. A miniature inductive loop probe (B-Dot probe) was employed to measure the radial magnetic field profile inside the discharge channel of the P5 with and without the plasma discharge. These measurements are accomplished with minimal disturbance to thruster operation with the High-speed Axial Reciprocating Probe system. The results of the B-Dot probe measurements indicate a change in the magnetic field topography from that of the vacuum field measurements. The measured magnetic field profiles are then examined to determine the possible nature and source of the difference between the vacuum and plasma magnetic field profiles. (C) 2002 American Institute of Physics. [DOI: 10.1063/1.1507771]
\end{abstract}

\section{INTRODUCTION}

The requirements of a spacecraft propulsion system for new mission profiles have increased recently beyond the current level of existing technology. An example of one recently prescribed requirement is for a propulsion system to operate in "dual-mode." Dual-mode is the ability of a thruster to operate efficiently in both a high-thrust and low-specific impulse mode (e.g., for orbit transfer operations) and a highspecific impulse and low-thrust mode (e.g., for station-keeping) ${ }^{1,2}$ Other design drivers for advanced space propulsion systems revolve around human space exploration. A piloted mission to Mars, for example, would require a reliable propulsion system with high power, high performance, and long life.

A favorable candidate for these types of missions is the closed-drift Hall thruster. A Hall thruster is a coaxial plasma device in which an applied magnetic field effectively traps electrons in the discharge channel of the thruster. The electrons are usually emitted from an external cathode while a magnetic circuit composed of electromagnetic solenoids and pole pieces typically creates the applied magnetic field. The magnetic circuit of a typical Hall thruster produces a radial magnetic field topography at the exit of the discharge channel with peak fields on the order of a few hundred-gauss. If the magnetic field topography in the discharge channel is designed properly, the accelerating ions will experience a focusing effect through what is referred to as a magnetic lens. ${ }^{3}$

One of the main characteristics of a Hall thruster is the azimuthally drifting electrons. These electrons form a region of azimuthal current in the discharge channel that is typically on the order of 5-10 times the discharge current. This estimate is based on Hall parameters expected in Hall thruster

${ }^{a)}$ Electronic mail: pypeters@engin.umich.edu discharge chambers; e.g., 200-300. ${ }^{4}$ This estimate can lead to Hall currents on the order of $150 \mathrm{~A}$ for a $5 \mathrm{~kW}$ Hall thruster.

Recent plasma measurements inside the discharge channel of the P5 as well as in other laboratory Hall thrusters indicate that the true, effective Hall parameter ranges from 10-20 near the anode to approximately 1000 in the acceleration region. ${ }^{5,6}$ These results suggest that the Hall current may be larger than previously thought, and that the induced magnetic field could impact the applied magnetic field topography significantly. As the magnitude of the Hall current increases at higher thruster power levels, the effects of the self-magnetic field induced by the Hall current may become important. As the self-magnetic field magnitude increases, the effect on the magnetic circuit applied field topography is greater, thus decreasing the chances of maintaining the desired magnetic lens profile in the discharge channel. In designing next-generation Hall thrusters, the magnetic field topography for a given magnetic circuit design can be predicted with great precision by using three-dimensional (3D) magnetostatic computer codes. However, to predict the magnetic field topography during thruster operation accurately, a better understanding of the effects of the magnetic field induced by the Hall current is needed.

The University of Michigan Plasmadynamics and Electric Propulsion Laboratory (PEPL) has endeavored in the past to develop and improve plasma diagnostic techniques to be used on plasma propulsion systems. ${ }^{7-14}$ An attempt to map the magnetic field topography near the exit of a D-55 Anode Layer Thruster (TAL) was performed at PEPL. ${ }^{14}$ The D-55 magnetic field measurements were limited to $15 \mathrm{~mm}$ downstream of the exit plane due to the large perturbations in thruster discharge current that resulted when the Hall probe was moved closer to the thruster. It was concluded that the 
Hall probe was entering the Hall current region and thus disrupting the stable operation of the thruster. To continue the research effort initiated by the D-55 very-near-field investigation, and to gather knowledge on the effects of the self-induced magnetic field at high power operation for a Stationary Plasma Thruster (SPT), a miniature inductive loop probe (B-Dot probe) system was developed and used in conjunction with the PEPL High-speed Axial Reciprocating Probe (HARP) system to map the radial magnetic field of the P5 Hall thruster.

The University of Michigan's HARP system was designed to address concerns associated with placing probes within the plasma of an operating Hall thruster. These concerns include probe life and thruster perturbation. The HARP system provides a unique opportunity to incorporate a time response B-Dot probe by virtue of its motion. B-Dot probes are typically used for pulsed and inductive plasma discharges with time-varying magnetic fields. ${ }^{15-17}$ However, by combining the high speed characteristics of the HARP table $(d x / d t)$ with the B-Dot probe response to a time-varying magnetic field $(d B / d t)$, one can measure a change in a magnetic field magnitude $(d B / d x)$ as the probe is swept into the discharge channel of the thruster.

This article will briefly describe the experimental facilities, the Hall thruster, and the HARP system. The theory of inductive loop operation is reviewed, as are the construction, set-up, and calibration of the B-Dot system. The thruster magnetic field profiles both in vacuum and in the presence of the plasma discharge are presented and discussed. Finally, several avenues are investigated to examine the nature of the observed change in the magnetic field profiles between the vacuum and plasma conditions.

\section{EXPERIMENTAL APPARATUS}

\section{A. Vacuum facilities}

All the experiments were conducted in the University of Michigan's Large Vacuum Test Facility (LVTF), which has a diameter of 6 meters and a length of 9 meters. Two 2000 cubic feet per minute (CFM) blowers and four 400 CFM mechanical pumps evacuate the LVTF chamber to moderate vacuum (30-100 mTorr). To reach high vacuum, the LVTF is equipped with seven CVI TM-1200 cryopumps, with a combined pumping speed of $\sim 500000 \mathrm{l} / \mathrm{s}$ on air, and $\sim 240000$ 1/s on xenon. The cryopump system can be operated with any number of pumps in use. For the experiments reported here the LVTF was operated with only four cryopumps to match the operating conditions of prior experiments. ${ }^{5,18}$ At the four-cryopump configuration, the combined pumping speed of $140000 \mathrm{l} / \mathrm{s}$ on xenon with a base pressure $1.6 \times 10^{-7}$ Torr was achieved. At an anode mass flow rate of $10.2 \mathrm{mg} / \mathrm{s}$ and a cathode mass flow rate of $0.6 \mathrm{mg} / \mathrm{s}$, the operating pressure of the LVTF was 1.1 $\times 10^{-5}$ Torr (xenon). The tank pressure during Hall thruster operation at $1.6 \mathrm{~kW}$ was $6.6 \times 10^{-6}$ Torr (xenon). The Hall thruster was mounted in the center of the vacuum chamber on an X-Y computer-controlled linear positioning system.

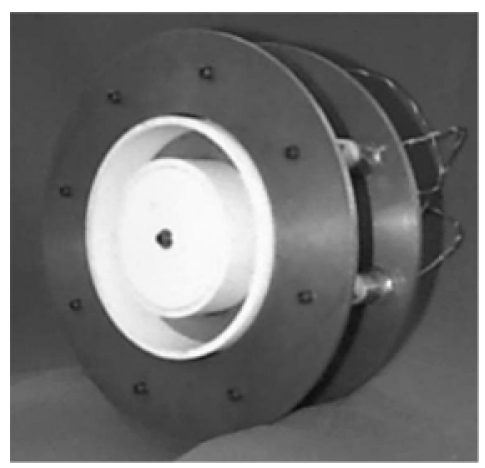

FIG. 1. P5, $5 \mathrm{~kW}$ class laboratory model Hall thruster.

\section{B. Hall thruster}

The experimental results presented in this paper were conducted on the $5 \mathrm{~kW}$-class laboratory model P5 Hall thruster. The P5 Hall thruster was developed by the University of Michigan and the Air Force Research Laboratory to serve as a test-bed for new diagnostics and for investigating Hall thruster processes. Depicted in Fig. 1, the P5 has an outer diameter of $170 \mathrm{~mm}$, a channel width of $25 \mathrm{~mm}$, and a channel depth of $38 \mathrm{~mm}$.

The P5 performance characteristics, presented in a previous work, ${ }^{19}$ are comparable to commercially available 5 $\mathrm{kW}$ thrusters. Table I shows the measured performance characteristics of the P5 for the two operating conditions used in this experiment. The hollow cathode used for this test was provided by the Moscow Aviation Institute (MAI). The cathode provides thermally emitted electrons to the discharge by a small disk of lanthanum hexaboride (LaB6).

\section{High-speed axial reciprocating probe}

The HARP system consists of a LM1210 high-speed linear motor and encoder manufactured by Trilogy. The linear encoder provides a linear resolution of 5 microns to a Pacific Scientific SC950 digital brushless servo drive controller. A computer controls the position, speed, acceleration, deceleration, and sweep configuration for the HARP system. The linear table is placed within a stainless steel shroud encased with graphite plates to protect it from the plume of the Hall thruster.

The primary issue involving internal probe measurements of a Hall thruster is the ability of the probe to survive in the presence of the discharge plasma and Hall current. Ablation of probe material also affects thruster operation. Therefore, the main driving factor in determining the maxi-

TABLE I. P5 thruster performance ranges for the experiments discussed in this work (Ref. 19).

\begin{tabular}{|c|c|c|c|c|c|}
\hline Case & $\begin{array}{l}\mathrm{V}_{\mathrm{d}} \\
(\mathrm{V})\end{array}$ & $\begin{array}{l}\mathrm{I}_{\mathrm{d}} \\
(\mathrm{A})\end{array}$ & $\begin{array}{c}\text { Power } \\
(\mathrm{kW})\end{array}$ & $\begin{array}{c}\text { Total } \\
\text { specific } \\
\text { impulse } \\
\text { (s) }\end{array}$ & $\begin{array}{c}\text { Total } \\
\text { efficiency }\end{array}$ \\
\hline (1) & 300 & 5.4 & 1.6 & 1550 & $48 \%$ \\
\hline (2) & 300 & 10.1 & 3.0 & 1650 & $51 \%$ \\
\hline
\end{tabular}


mum resonance time that a probe can remain in the discharge channel of a thruster is the characteristic time for probe material ablation. The ablation time for $99 \%$-pure alumina has been determined to be approximately $150 \mathrm{~ms}$ for a $5 \mathrm{~kW}$ plasma discharge. ${ }^{5}$ The time that the B-Dot probe spent in the discharge channel for these experiments was approximately $80 \mathrm{~ms} \pm 10 \mathrm{~ms}$. The peak velocity and acceleration of the HARP for these experiments was approximately $\pm 5.5 \mathrm{~m} / \mathrm{s}$ and +130 to $-100 \mathrm{~m} / \mathrm{s}^{2}$, respectively.

Another important issue is the actual heating of the probe during the sweep into the discharge plasma. If the probe resonance time in the discharge plasma is significantly long to allow the probe to heat up, thus changing the electrical characteristics of the probe's circuit, the results of the mapped magnetic field profile for the plasma case may differ from that of the vacuum measurements. Employing the same method for estimating the amount of time before ablation of the probe material in a $5 \mathrm{~kW}$ plasma discharge, ${ }^{5}$ one can determine a bulk heating of a probe of a given size, material, discharge plasma parameters, and exposure time. It was determined analytically that the maximum increase in temperature for a typical ceramic probe used during this investigation will be approximately 1.5 degrees per sweep. The calculated temperature increase of the probe in the discharge plasma was relatively low; therefore no experimental measurements of the probe temperature characteristics were attempted.

\section{Inductive loop probe}

The inductive loop probe is a well-established plasma diagnostic technique for time-varying magnetic fields. Typical plasma discharges that make use of the B-Dot probing technique include pulsed and inductive plasmas. ${ }^{15-17}$ The basic operating principle of the B-Dot probe is based on the observation that current is induced in a conducting coil immersed within a time-varying magnetic field. The ensuing output voltage from the coil is proportional to its crosssectional area $(A)$, the number of turns in the coil $(n)$, and the time characteristic of the magnetic field $(d B / d t)$. The expression of the voltage output from a B-Dot probe is given as Eq. (1)

$$
V=n A d B / d t \text {. }
$$

Since Hall thrusters typically operate with a steady applied magnetic field, direct application of the B-Dot probe is problematic. To circumvent this issue, the HARP position system was used to provide a time-varying magnetic field signal by virtue of moving the probe into (and out of) the applied, steady magnetic field. Therefore, by combining the motion of a high-speed table $(d x / d t)$ with the integrated signal of a B-Dot probe in a time-varying magnetic field $(d B / d t)$, one is able to measure the DC applied magnetic field as the probe is swept into and out of the Hall thruster discharge channel.

A dual-supply integrator that incorporates an Analog Devices AD549 ultra-low input bias current operational amplifier and an AD624 precision instrumentation amplifier was

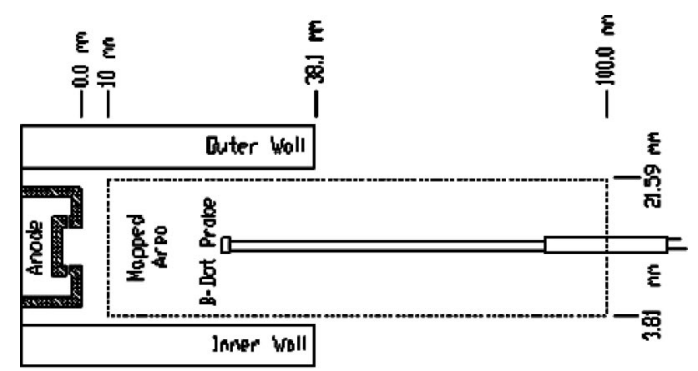

FIG. 2. B-Dot probes mapped area inside and outside of the P5 discharge channel.

chosen to integrate the B-Dot probe raw signal. The expression of the integrated output voltage from a B-Dot probe is shown in Eq. (2)

$$
V=\frac{n A}{R C G} B,
$$

where the $G$ is the amplifier gain, $R C$ is the integrator resistance and capacitance, respectively, and $B$ is the magnetic field strength. The amplified voltage output from the B-Dot probe integrator circuit was recorded with a Tektronix TDS 540 digital oscilloscope in high-resolution acquisition mode. The data were then downloaded to a computer for processing.

The B-Dot probe support structures used for this investigation were constructed of $99 \%$ alumina ceramic tubes and high-temperature ceramic paste. A number of probe configurations were built evaluated for this effort. The inductive loops were wound with 38 and 40 gauge magnetic wires with enamel nonconductor coatings. The coils used in this investigation were wound around $1.6-2.5 \mathrm{~mm}$ diameter alumina ceramic tubes $2.2-2.5 \mathrm{~mm}$ in length. The number of turns in the probe coils ranged from 89 to 136 . The average data collection area of the probes used in this investigation was $3.3 \mathrm{~mm}^{2}$ over an average length of $2.35 \mathrm{~mm}$. The final dimensions of the B-Dot probes, including a protective layer of 99\% alumina ceramic, used in this investigation ranged from 4 to $4.3 \mathrm{~mm}$ in diameter and $4-4.2 \mathrm{~mm}$ in length.

Figure 2 shows the mapped area inside the discharge channel of the P5 thruster. The B-Dot probe was axially swept from $150 \mathrm{~mm}$ downstream of the exit plane to $10 \mathrm{~mm}$ downstream of the anode. This axial sweep profile was repeated for three radial positions, $3.81 \mathrm{~mm}$ from the inner wall, the centerline (at $12.7 \mathrm{~mm}$ ), and $3.81 \mathrm{~mm}$ from the outer wall of the discharge channel. In an attempt to minimize the heating of B-Dot probe between sweeps, the probe home position was set to $152 \mathrm{~mm}$ downstream of the thruster exit plane. The cathode plane was located $50 \mathrm{~mm}$ downstream of the thruster exit plane and was oriented $45^{\circ}$ counter-clock wise from the plane mapped. The results presented in this paper will cover the first $100 \mathrm{~mm}$ downstream of the anode. 


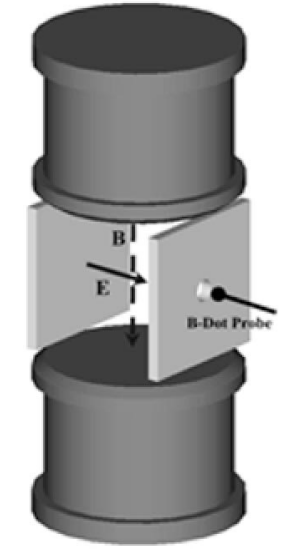

FIG. 3. The $\mathbf{E} \times \mathbf{B}$ source configuration.

\section{RESULTS AND ANALYSIS}

\section{A. Calibration of B-Dot probe}

The initial goal of this experiment was to calibrate the B-Dot probe and integrator circuit with an electric and magnetic field $(\mathbf{E} \times \mathbf{B})$ source. The idea behind the $\mathbf{E} \times \mathbf{B}$ source was to approximate, under a controlled and understood manner, the fields that the B-Dot probe would experience when the probe was traveling through the discharge channel of an operating thruster. The $\mathbf{E} \times \mathbf{B}$ source was comprised of two electromagnetic coils, to provide the magnetic field, and two parallel plates for the electric field. The configuration of the $\mathbf{E} \times \mathbf{B}$ source can be seen in Fig. 3 .

The magnetic field of the $\mathbf{E} \times \mathbf{B}$ source was first mapped using a NIST-traceable Walker Scientific MG-5DAR Hall probe. Then the B-Dot probe and HARP systems were configured to measure the $\mathbf{E} \times \mathbf{B}$ source with the applied magnetic field, or the applied magnetic field with a static or timevarying electric field. A time-varying electric field was simulated to approximate the oscillations of the discharge plasma in a Hall thruster during normal operation. All parameters of the B-Dot probe and HARP, such as speed, acceleration, deceleration, probe sweep length, and transmission line length that would be used during experiments with the P5 were used in the calibration runs.

Figure 4 shows the measured magnetic field profile of the $\mathbf{E} \times \mathbf{B}$ source as measured by the B-Dot and Hall probes at atmospheric pressure with and without an applied static or

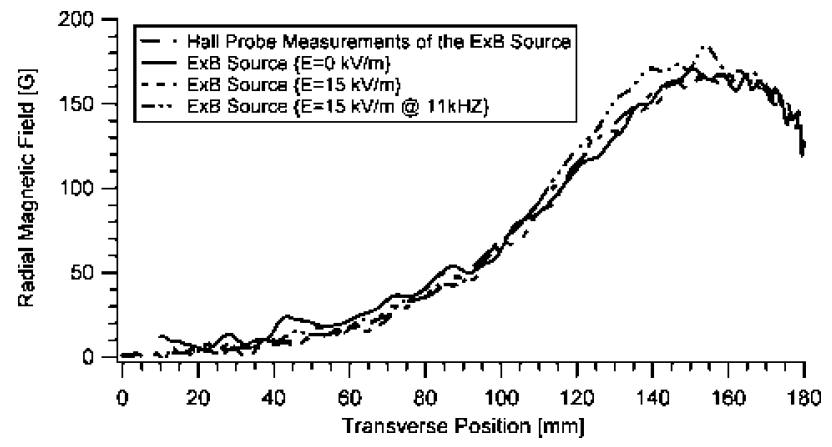

FIG. 4. B-Dot and Hall probe measurements of the $\mathbf{E} \times \mathbf{B}$ source at atmospheric pressure $\left\{I_{\text {Coil }}=9 \mathrm{~A}\right\}$. time-varying $(11 \mathrm{kHz})$ electric field of $15 \mathrm{kV} / \mathrm{m}$. The peak electric field, which resides in a small region of the discharge chamber at the onset of the acceleration zone, can reach 25 $\mathrm{kV} / \mathrm{m}$ at 300 volts and $5.4 \mathrm{~A}$, and $20 \mathrm{kV} / \mathrm{m}$ at 300 volts and $10 \mathrm{~A}$ according to the internal emissive probe measurements. ${ }^{5,18}$ The $15 \mathrm{kV} / \mathrm{m}$ that was used during the calibration of the B-Dot probes should still indicate if the B-Dot probes are influenced by the presence of a steep steady or time-varying electric field. The measured data presented in Fig. 4 are composed of five sets of B-Dot traces averaged together at the same $\mathbf{E} \times \mathbf{B}$ source input settings. Figure 4 also contain the Hall probe traces of the $\mathbf{E} \times \mathbf{B}$ source at the two investigated conditions. As can be seen in Fig. 4, the B-Dot probe captured the profile and magnitude of the $\mathbf{E} \times \mathbf{B}$ source. The presence of the $15 \mathrm{kV} / \mathrm{m}$ static and time-varying electric fields had no noticeable influence on the measured magnetic field from the B-Dot probe.

\section{B. Vacuum field measurements with B-Dot probe}

The vacuum magnetic field profiles of the P5 thruster were mapped using the B-Dot and HARP systems along the radial and axial positions described earlier. Two thruster power levels were investigated; 1.6 and $3.0 \mathrm{~kW}$, both at a discharge voltage of $300 \mathrm{~V}$. The inner and outer electromagnetic coils were operated in a manner that minimized the discharge current of the thruster. Once these coil currents were determined for both power levels, the P5 discharge was extinguished while the magnets were left on for vacuum field measurements.

The B-Dot and Hall probe vacuum field measurements of the P5 at coil current settings for 1.6 and $3.0 \mathrm{~kW}$ are shown in Figs. 5 and 6, respectively. The thruster exit plane and the mid-plane of the magnetic circuit outer and inner front poles are labeled in these figures. Once again, each B-Dot profile presented represents the average of 5 or more data sweeps. All trends observed in individual B-Dot sweeps are captured in the average profile. Figures 5 and 6 show that magnetic field profiles measured with the B-Dot probe match those of the NIST-traceable Hall probe for both coil settings.

\section{Magnetic field measurements with discharge}

The discharge channel radial magnetic field with the P5 thruster operating at 1.6 and $3.0 \mathrm{~kW}$ was mapped at three radial positions. The $\mathrm{P} 5$ was operated for a minimum of forty minutes before B-Dot sweeps were made to allow the engine to reach thermal equilibrium; i.e., when the electromagnetic coil voltages of the magnetic circuit stabilized. This step was conducted to ensure that any anomalies measured with the B-Dot probe were not due to thermal expansion of the magnetic circuit components throughout the duration of the experiment. The results of the B-Dot probe sweeps for the 1.6 $\mathrm{kW}$ thruster power level (300 volt, 5.4 A) are shown in Fig. 7. Figure 7 also contains a plot of the thruster discharge current as a function of probe position and the corresponding bench top Hall probe magnetic field measurements. As can be seen in Fig. 7, the magnitude and profile of the magnetic field in the discharge channel of the P5 Hall thruster was influenced by the presence of the plasma discharge. 

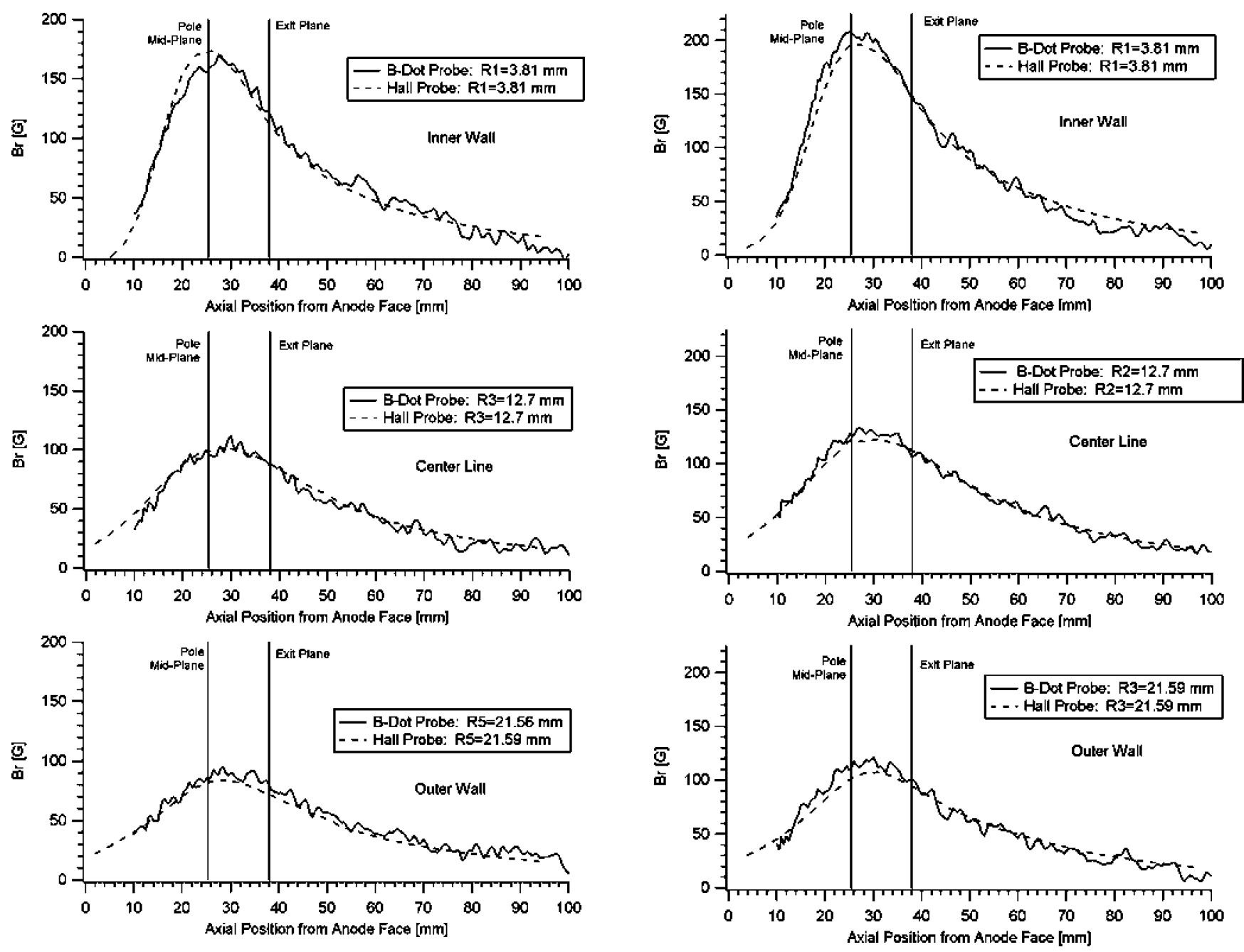

FIG. 5. Vacuum magnetic field profiles of the P5 at $1.6 \mathrm{~kW}\left\{I_{\mathrm{in}}=2 \mathrm{~A}\right.$, $\left.I_{\text {out }}=1 \mathrm{~A}\right\}$.

The B-Dot probe results for a $3 \mathrm{~kW}$ plasma, the vacuum Hall probe measurements, and thruster discharge current data at three different radial positions for the $3.0 \mathrm{~kW}$ power level are shown in Fig. 8. Once again the B-Dot profiles with the thruster operating at $3.0 \mathrm{~kW}$ differ from those in vacuum at the identical magnet settings. There are several possible explanations to account for these measured differences in the magnetic field profiles. The first and foremost explanation, assuming that the B-Dot probe is functioning properly, is the effect of the azimuthal electron drift (Hall current) self magnetic field. As discussed earlier, the Hall current of a closeddrift thruster can be 5-10 times the discharge current ${ }^{4}$ or in light of recent internal plasma parameter measurements, ${ }^{5}$ possibly much higher.

In both the $1.6 \mathrm{~kW}$ and the $3.0 \mathrm{~kW}$ cases we see that the B-Dot probe magnetic field profiles with the discharge plasma closely match the vacuum field profiles along the inner wall of the discharge channel. However, the profiles are significantly different near the outer wall of the discharge channel and particularly along the center of the channel. The largest difference in the profiles occur slightly downstream of the thruster exit plane. In the $1.6 \mathrm{~kW}$ results, there is a spike in the measured magnetic field $10 \mathrm{~mm}$ inside of the

FIG. 6. Vacuum magnetic field profile of the P5 at $3.0 \mathrm{~kW}\left\{I_{\mathrm{in}}=3 \mathrm{~A}\right.$, $\left.I_{\text {out }}=2 \mathrm{~A}\right\}$.

discharge channel. A characteristic spike exists in the $3.0 \mathrm{~kW}$ data as well; however, in this case the spike is $7 \mathrm{~mm}$ inside the thruster channel. Neither of these spikes nor the large increases in the B-Dot magnetic field profiles corresponds to locations of peak electric fields in the thruster. ${ }^{5}$ The peak electric fields of the P5 are located approximately $5 \mathrm{~mm}$ inside the discharge channel for the $1.6 \mathrm{~kW}$ thruster power level and approximately $2 \mathrm{~mm}$ inside the channel for the 3.0 $\mathrm{kW}$ power level ${ }^{18}$ (data not shown). Thruster discharge current was recorded as the probe entered the discharge channel for the two power conditions investigated and are shown in Figs. 7 and 8. The maximum disturbance to the thruster discharge current was $8 \%$; during the centerline sweep at the $1.6 \mathrm{~kW}$ power level. The axial region where the greatest perturbation in the thruster discharge current was recorded corresponds to a drop in the measured magnetic field profiles for each of the three radial sweeps for the $3.0 \mathrm{~kW}$ test condition. This can also be seen in the outer wall sweep of the $1.6 \mathrm{~kW}$ case. A possible explanation for these features is that the probe is blocking the natural path of the Hall current in the thruster channel. This explanation can also be argued by the greater perturbation to the thruster discharge current for the centerline and outer wall sweeps, since this region of the 

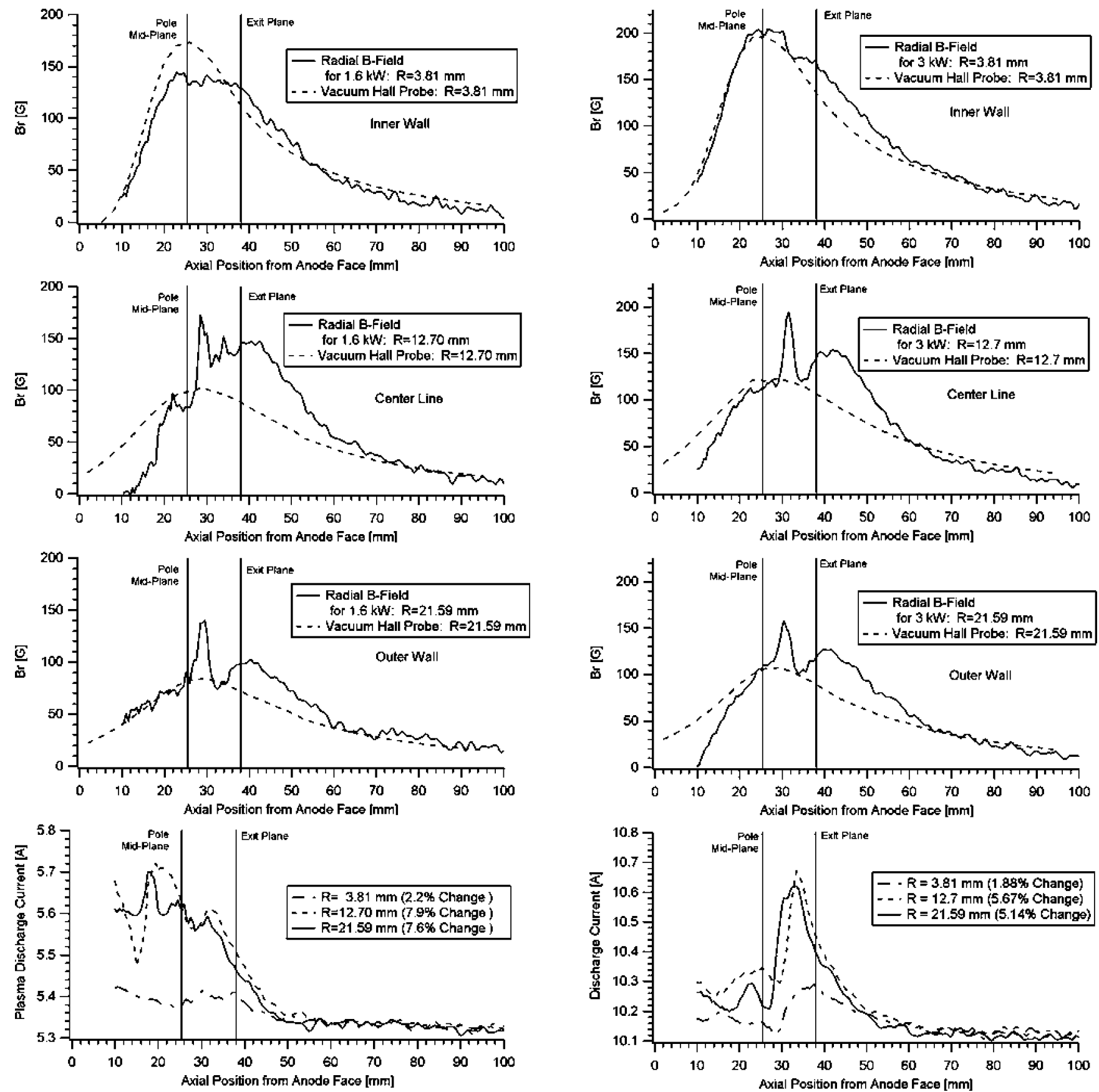

FIG. 7. B-Dot probe magnetic field data at $1.6 \mathrm{~kW}$ and thruster discharge current as a function of probe position.

discharge channel is where most of the Hall current is for the P5 thruster. ${ }^{5}$

Another possible explanation, though less likely, for the observed disturbances in the discharge current is thermal expansion of the magnetic circuit. However, this prospect was investigated by measuring the vacuum field profiles of the thruster before and immediately after the thruster was operated for an extended amount of time. No discernable changes in the vacuum field profiles were observed during the analysis of the data (not shown).

One final possibility for the measured difference in the results from the vacuum and plasma discharge profiles is that the B-Dot probe output voltage is perturbed by the high elec-

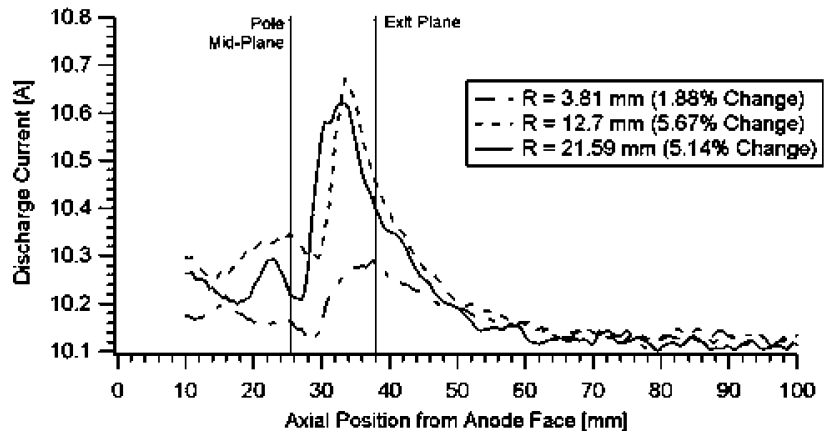

FIG. 8. B-Dot probe magnetic field data at $3.0 \mathrm{~kW}$ and thruster discharge current as a function of probe position.

tric field in the discharge channel of the thruster. Several precautions were addressed during the fabrication and calibration of the B-Dot probe system. The first was to place the integrator circuit as near as possible to the probe, which meant that the integrator was located in the shroud that covers the HARP table. There was no indication, from the baseline sweeps of the HARP table and the B-Dot system, of electrostatic interference in the probe or the circuit from the operation of the HARP table alone. Another precaution addressed was to add a low resistance to the output of the B-Dot probe through the selection of the transmission lines and circuit design, thus effectively providing the B-Dot probe with a voltage divider for any unavoidable electro- 
static pickup. ${ }^{15}$ The final precaution was to calibrate the B-Dot system with an $\mathbf{E} \times \mathbf{B}$ source as described earlier. It was shown in Fig. 4 that as the B-Dot probe swept the $\mathbf{E}$ $\times \mathbf{B}$ source with either a known applied magnetic, static or time-varying electric fields that there was no indication of the probe being affected by the presence of a large electric field.

\section{DISCUSSION}

To understand the data presented in this paper several avenues were investigated. The first approach was to make use of recent Hall parameter calculations inside the discharge chamber of a Hall thruster, ${ }^{5,6}$ which determined that the Hall parameter could be as high as 1000 at the exit of the discharge channel. The Hall parameter is defined as the ratio between the azimuthal electron current (Hall current, $I_{\text {Hall }}$ ) and transverse electron current $\left(I_{\mathrm{ez}}\right)$ and the ratio of the transverse current region $\left(A_{\mathrm{ez}}\right)$ to the Hall current region $\left(A_{\text {Hall }}\right)$ as seen in the following expression:

$$
\Omega=\frac{j_{\text {Hall }}}{j_{e z}}=\frac{I_{\text {Hall }}}{I_{e z}} \frac{A_{e z}}{A_{\text {Hall }}} .
$$

The axial electron current ranges from 25 to 30 percent of the discharge current ${ }^{20}$ for the P5 Hall thruster. Assuming the majority of the Hall current resides in a region near the exit of the discharge channel and encompasses the width of the channel, and given a Hall parameter of 1000, we find that the Hall current is on the order of $90 \mathrm{~A}$. A simple calculation for a self magnetic field, induced by the Hall current, can be made using the Biot-Savart law for a current flowing in a long straight wire. This first-order calculation yields an induced self-field of approximately 15 Gauss $7 \mathrm{~mm}$ downstream of the center of the prescribed Hall current region.

The first order calculation of the self-field does not express the overall mechanism of the induced magnetic field profile in a Hall thruster. The Hall thruster applied magnetic field topography is a consequence of the design of the system's magnetic circuit. It is known that changing one component of the magnetic circuit (e.g., screen length, thickness, or width) could lead to considerable modification to the magnetic field topography in the channel of a thruster. ${ }^{21}$ To address the concerns of a self-field influence on the applied magnetic field, a 3D magnetostatic simulation using Magnet6 by Infolytica was performed. Figure 9 is an illustration of the 3D model used in the simulation.

The model of the P5 Hall thruster, presented in Fig. 9, was created to simulate the influence that the Hall current self-field has on the applied magnetic field from the region 10-100 mm downstream of the anode. The area of investigation continued past the exit of the discharge channel since it has been shown that a good portion of the ion acceleration occurs past the thruster exit. ${ }^{5,22}$ Therefore, this region was investigated as another source of self-field for the thruster. The 3D model also took into account the influence of the induced self-fields from each Hall current region on a single point of interest. The Hall current for this simulation was determined in a similar manner as in the first order calculation presented above, except five sets of Hall parameter data

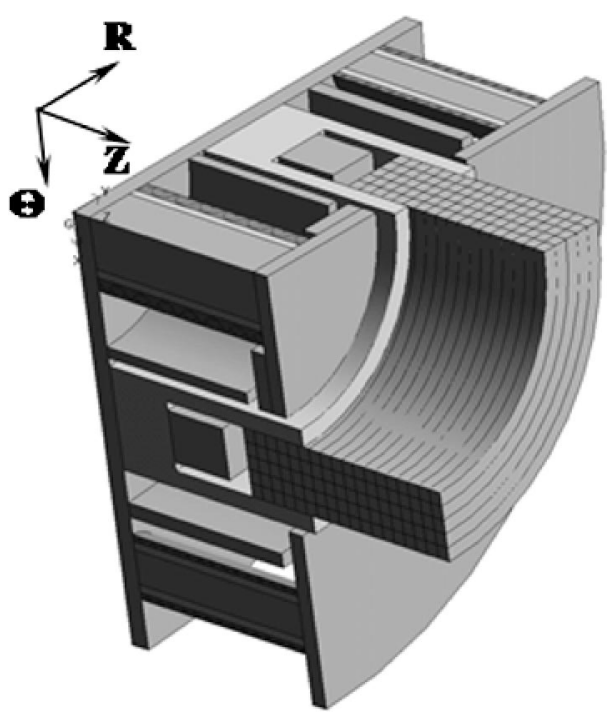

FIG. 9. The 3D quarter model used in Magnet6 to simulate P5 self-fields.

across the channel width and ranging from 10 to $100 \mathrm{~mm}$ downstream of the anode was used. To reduce the computational time and model complexity, the calculated Hall current profiles were interpolated to 90 regions, approximately $5 \mathrm{~mm}$ by $5 \mathrm{~mm}$ each, as seen in Fig. 9. The sum current for all the Hall current regions inside and outside of the discharge channel equated to a total Hall current of $156 \mathrm{~A}$, which was different than the predicted 27-54 amps for the P5 operating at $5.4 \mathrm{~A}^{4}{ }^{4}$ The ratio of the Hall to the discharge currents for the calculated Hall current above was $I_{\mathrm{Hall}} / I_{d}=28.8$. This ratio indicated that the average Hall parameter for the P5 thruster, for the 90 regions method, was approximately 478 $(\approx 478)$. The main difference in the Hall parameter for the P5, as compared to the value given by Kim, ${ }^{4}$ was that the calculation of the Hall parameter accounted for the ion acceleration that occurs downstream of the exit plane. Figure 10 illustrates the axial profiles of the calculated Hall current for five radial sweeps across the discharge channel and the sum of the Hall current as a function of axial position. The self-field modeling results for the $300 \mathrm{~V} 5.4$ A thruster discharge condition is presented in Fig. 11.

As it can be seen in Fig. 11, the model predicts a change in the radial magnetic field profile along the center of the

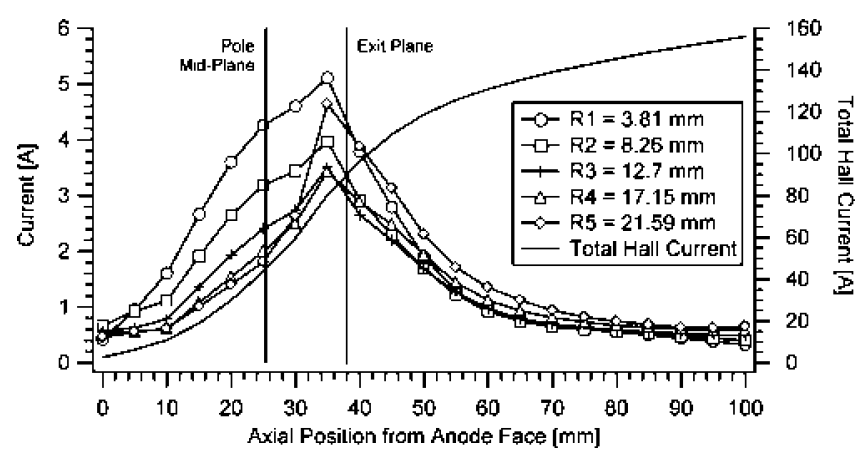

FIG. 10. The calculated Hall current radial profiles across the discharge channel and the sum of the profiles as a function of axial position (note that the thruster exit is at $38 \mathrm{~mm}$ ). 


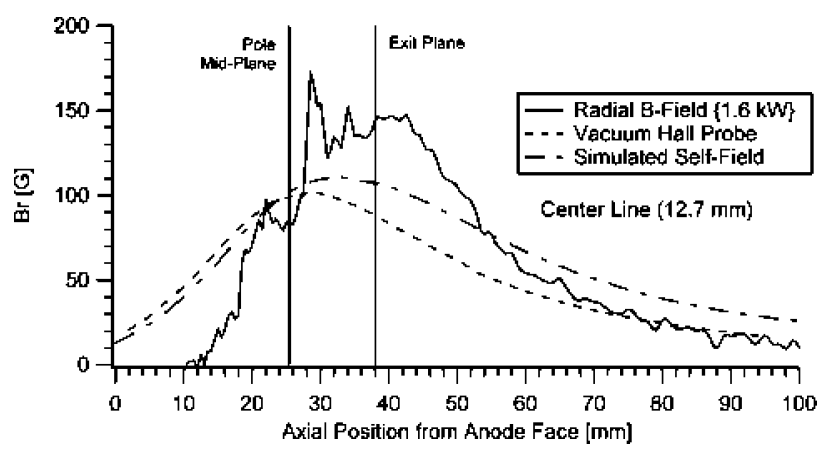

FIG. 11. The simulated self-field for the P5 operating at $1.6 \mathrm{~kW}$.

discharge channel. Similar results are obtained for the $300 \mathrm{~V}$ and $10 \mathrm{~A}$ thruster condition (not shown). However, these observed changes are not as large as the measured field profiles from the B-Dot probe. This could possibly be due to the technique used to calculate the Hall current from the Hall parameters, a problem with the calculated Hall parameters, or the B-Dot probe measurements.

Another issue that has not been discussed in this paper, so far, is the possibility of a dielectric sheath forming around the probe. ${ }^{23,24}$ Since the probe is constructed of a dielectric material and is inserted into the discharge plasma of a thruster, the azimuthal electron current would dominate the collisions with the probe's surface thus establishing a negative sheath that might redirect the Hall current around the probe. The threshold electron energy for the establishment of an electron-repellant sheath for alumina has been measured to be approximately $20 \mathrm{eV} .{ }^{24}$ According to the measurements by Haas in Ref. 5 the peak electron drift energy for the 1.6 $\mathrm{kW}$ case was upwards of $25 \mathrm{eV}$ and the observed electron thermal energy had a maximum of $40 \mathrm{eV}$. The presence of a negative sheath encompassing the probe could increase the electron current density to ether side of the probe, thereby changing the normal Hall current profile, and therefore, the local magnetic field. The dielectric sheath thickness is on the order of a Debye length, ${ }^{25}$ which is approximately $0.05 \mathrm{~mm}$ for the internal plasma parameters of the P5 thruster operating a $1.6 \mathrm{~kW} .^{5}$ The sheath thickness is not large compared to the size of the probe, but the probe and the thin sheath could sway the profile of the Hall current. A Magnet6 model was created to test the possibility of this modified Hall current. The results from the model (data not shown) did not indicate a significant change in the self-field radial magnetic field profile as seen in Fig. 11. A final issue with a dielectric probe is that it could be absorbing high-energy electrons and reemitting low temperature electrons through secondary electron emission. ${ }^{26}$ According to Ref. 24 the maximum secondary electron emission coefficient for alumina is 4.8 for electrons impacting at normal incidence and at $1300 \mathrm{eV}$. At the drift and thermal energies of the electrons measured in the P5 thruster $(25-40 \mathrm{eV})$, the general trend is for the secondary electron emission coefficient to be less than unity. ${ }^{24}$ The formation of a negative sheath around the probe would prohibit a majority of the primary electrons from reaching the probe and thereby limit the influence of secondary electron emission.
The predicted thrust, for a Hall thruster, can be obtained analytically from the magnetohydrodynamic (MHD) momentum equation shown in Eq. (4) and knowing the azimuthal Hall current $\left(j_{\text {Hall }}\right)$ in a given volume $(V)$ and the radial magnetic field $(\mathrm{Br})$ :

$$
\mathbf{F}=-\nabla P+\rho_{c} \mathbf{E}+\mathbf{j} \times \mathbf{B} .
$$

Assuming that the electron pressure term $(P)$ is small ${ }^{5}$ and a quasineutral plasma $(c=0)$, Eq. (4) reduces to the following $\mathbf{j} \times \mathbf{B}$ force.

$$
F_{y}(r, z)=j_{\text {Hall }}(r, z) B_{r}(r, z) V(r, z) .
$$

Equation (5) can be used to calculate the thrust in each individual Hall current region, as described above, and then the total thruster can be found by summing all the regions. The calculated thrust for the Hall current shown in Fig. 10 using Eq. (5) and the measured radial magnetic field profiles from the B-Dot probe at the $1.6 \mathrm{~kW}$ plasma case is approximately $300 \mathrm{mN}$, which is much greater than the measured thrust of $98 \mathrm{mN} .{ }^{19}$ When the Hall current density profiles from Ref. 5 are combined with the vacuum magnetic fields shown on Fig. 5, the resulting thrust predicted is $105 \mathrm{mN}$. The conclusion of this exercise is that a Hall current that is significantly large enough to produce the self-field observed in the B-Dot probe traces would equate to a thrust that is much larger than what is measured. However, other investigations have uncovered evidence that the Hall current may be significant in some cases. One such investigation involved the magnetic field integration impact testing of a D-55 TAL by NASA GRC for the RHETT2/EPDM program. ${ }^{27}$ The magnetic dipole measured $1.28 \mathrm{~m}$ upstream of the thruster exit plane was observed to increase by $22 \%$ above the vacuum applied field during thruster operation at $300 \mathrm{~V}$ and $2.5 \mathrm{~A}$. A Hall current of $50 \mathrm{~A}$ is required to produced the observed change in the axial magnetic field. ${ }^{28}$ However, a Hall current of this magnitude would produce a thrust of 86 $\mathrm{mN}$, which is twice the measured thrust of $43.2 \mathrm{mN} .^{29}$ Clearly, further investigations are needed before our understanding of the role that the Hall current plays in establishing the Hall thruster magnetic field topography becomes complete.

\section{SUMMARY}

The magnetic field topography of the P5 Hall thruster was successfully mapped during operation of the thruster with a plasma discharge. The experimentally mapped magnetic field profiles along the width of the discharge channel of a Hall thruster deviated from the vacuum field profile, previously measured with a Hall probe. The largest increase observed was approximately $70 \mathrm{G}(70 \%)$ on the centerline sweep of the $1.6 \mathrm{~kW}$ operation condition. The Hall current of the P5 was then calculated from previously determined Hall parameters and incorporated into a 3D magnetostatic model to estimate the influence of the Hall current self-magnetic field on the applied magnetic field topography. The $\mathbf{j} \times \mathbf{B}$ forces were then solved to establish if the Hall current required to influence the magnetic field topography, as observed in the B-Dot probe measurements, yields engine 
thrust levels that are consistent with those measured. The calculated thrust values from the $\mathbf{j} \times \mathbf{B}$ force were 2 to 3 times the actual measured values at the given operating parameters. Several experiments and calculations were conducted to test the B-Dot probe system reliability under the plasma conditions of a typical Hall thruster discharge. The experiments and calculations did not demonstrate any conclusive evidence of a flaw in the design or methodology utilized for measuring the magnetic field discussed in this paper. Furthermore, the topic was complicated by documentation of independent nonintrusive magnetic dipole measurements of the D-55 TAL at NASA GRC. The magnetic dipole measurements of the D-55 show a $22 \%$ increase of the measured magnetic dipole when the discharge was ignited. It is clear that a better understanding of the influence of a Hall thruster self-field is required if higher-power thrusters are to be developed.

\section{ACKNOWLEDGMENTS}

The authors would like to acknowledge the support of this research by NASA through Contract No. NGT3-52348 and NAG3-2307 (Robert Jankovsky, technical monitor for both grants) and the AFOSR through Contract No. F4962001-1-0061 (Dr. Mitat Birkan is the technical monitor). The authors would also like to thank the Air Force Research Laboratory for their assistance in the design and fabrication of the P5 Hall thruster, Dr. Sergi Khartov at MAI for the use of the LaB6 cathode, and the graduate students at PEPL for their assistance in setting up and running the experiments for this investigation. The authors would also like to thank the technical support staff of the Aerospace Engineering Department.

${ }^{1}$ G. W. Butler, J. L. Yuen, S. O. Tverdokhlebov, A. V. Semenkin, A. V. Kochergin, A. E. Solodulthin, and R. S. Jankovsky, Proceedings of the 2000 AIAA Joint Propulsion Conference, AIAA Paper No. 00-3254 (American Institute of Aeronautics and Astronautics, Washington, DC, 2000).

${ }^{2}$ S. R. Oleson, Proceedings of the 2000 AIAA Joint Propulsion Conference, AIAA Paper No. 00-3413 (American Institute of Aeronautics and Astronautics, Washington, DC, 2000).

${ }^{3}$ V. M. Gavryshin, V. Kim, V. I. Kozlov, and N. A. Maslennikov, Proceedings of the 1995 International Electric Propulsion Conference, IEPC Paper No. 95-38 (Electric Rocket Propulsion Society, Columbus, OH, 1995).

${ }^{4}$ V. Kim, J. Propul. Power 14, 736 (1998).

${ }^{5}$ J. M. Haas and A. D. Gallimore, Phys. Plasmas 8, 652 (2001).

${ }^{6}$ N. B. Meezan and M. A. Cappelli, Proceedings of the 2000 AIAA Joint Propulsion Conference, AIAA Paper No. 00-3420 (American Institute of Aeronautics and Astronautics, Washington, DC, 2000).
${ }^{7}$ J. M. Haas, A. D. Gallimore, K. McFall, and G. Spanjers, Rev. Sci. Instrum. 71, 4131 (2000).

${ }^{8}$ G. J. Williams, T. B. Smith, F. S. Gulczinski, and A. D. Gallimore, J. Propul. Power 18, 489 (2002).

${ }^{9}$ B. E. Beal, J. M. Haas, and A. D. Gallimore, ICOPS 00-4A03 (2000).

${ }^{10}$ R. R. Hofer, J. M. Haas, and A. D. Gallimore, Proceedings of the 1999 International Electric Propulsion Conference, IEPC Paper No. 99-113 (Electric Rocket Propulsion Society, Columbus, OH, 1999).

${ }^{11}$ F. S. Gulczinski and A. D. Gallimore, J. Propul. Power 17, 418 (2001).

${ }^{12}$ A. D. Gallimore, J. Spacecr. Rockets 38, 441 (2001).

${ }^{13}$ L. B. King and A. D. Gallimore, Rev. Sci. Instrum. 68, 1183 (1997).

${ }^{14}$ M. T. Domonkos, A. D. Gallimore, C. M. Marrese, and J. M. Haas, J. Propul. Power 16, 91 (2000).

${ }^{15}$ R. H. Lovberg, Plasma Diagnostic Techniques (Academic, New York, 1965), Chap. 3.

${ }^{16}$ R. Piejak, V. Godyak, and B. Alexandrovich, J. Appl. Phys. 81, 3416 (1997)

${ }^{17}$ D. C. Black, "Direct Magnetic Field Measurements of Flow Dynamics and Micro-Turbulence Enhanced Electron Collisionality in Magnetized Coaxial Accelerator Channels," North Carolina State University Department of Nuclear Engineering, Ph.D. dissertation, 1996.

${ }^{18}$ J. M. Haas and A. D. Gallimore, Proceedings of the 1999 International Electric Propulsion Conference, IEPC Paper No. 99-078 (Electric Rocket Propulsion Society, Columbus, OH, 1999).

${ }^{19}$ J. M. Haas, F. S. Gulczinski, A. D. Gallimore, G. G. Spanjers, and R. A. Spores, Proceedings of the 1998 AIAA Joint Propulsion Conference, AIAA Papers No. 98-3503 (American Institute of Aeronautics and Astronautics, Washington, DC, 1998).

${ }^{20}$ R. R. Hofer and R. S. Jankovsky, Proceedings of the 2001 AIAA Joint Propulsion Conference, AIAA Paper No. 01-3322 (American Institute of Aeronautics and Astronautics, Washington, DC, 2001).

${ }^{21}$ R. R. Hofer, P. Y. Peterson, and A. D. Gallimore, Proceedings of the 2001 International Electric Propulsion Conference, IEPC Paper No. 01-036 (Electric Rocket Propulsion Society, Santa Fe, NM, 2001).

${ }^{22}$ G. J. Williams, T. B. Smith, F. S. Gulczinski, B. E. Beal, A. D. Gallimore, and R. P. Drake, Proceedings of the 1999 AIAA Joint Propulsion Conference, AIAA Paper No. 99-2424 (Institute of Aeronautics and Astronautics, Washington, DC, 1999).

${ }^{23}$ E. Ahedo, P. Martínez Cerezo, and M. Martínez-Sánchez, Proceedings of the 2001 AIAA Joint Propulsion Conference, AIAA Paper No. 01-3323 (Institute of Aeronautics and Astronautics, Washington, DC, 2001).

${ }^{24}$ S. C. Brown, Basic Data of Plasma Physics (American Institute of Physics Press, New York, 1994), Chap. 9.

${ }^{25}$ H. S. Uhm, J. Plasma Phys. 63, Part 2, 129 (2000).

${ }^{26}$ E. Y. Choueiri, Proceedings of the 2001 AIAA Joint Propulsion Conference, AIAA Paper No. 01-3504 (Institute of Aeronautics and Astronautics, Washington, DC, 2001).

${ }^{27}$ J. M. Sankovic, E. J. Pencil, and D. T. Jacobson, Proceedings of the 1997 International Electric Propulsion Conference, IEPC Paper No. 97-109 (Electric Rocket Propulsion Society, Columbus, OH, 1997).

${ }^{28}$ P. Y. Peterson, J. M. Haas, and A. D. Gallimore, Proceedings of the 2001 International Electric Propulsion Conference, IEPC Paper No. 01-030 (Electric Rocket Propulsion Society, Santa Fe, NM, 2001).

${ }^{29}$ J. M. Sankovic, D. H. Manzella, and M. F. Osborn, Proceedings of the 1997 International Electric Propulsion Conference, IEPC Paper No. 97102 (Electric Rocket Propulsion Society, Columbus, OH, 1997). 